\title{
Herramienta para la realización de auditorías internas para empresas con sistemas HSEQ*
}

\author{
Internal audit tool for companies with HSEQ systems
}

\section{Ferramenta para auditoria interna de empresas com sistemas de HSEQ}

Recibido: 26 de julio de 2019

Revisado: 22 de enero de 2020

Julián Chaparro Gonะále***

Icontec

René Fernando Martín Beltrán ${ }^{* * *}$

Icontec

Cómo citar este artículo: Chaparro, J., y Martín, R. F. (2020). Herramienta para la realización de auditorías internas para empresas con sistemas HSEQ. Signos, Investigación en Sistemas de Gestion, 12(2), 47-57. Dor: https://doi.org/10.15332/24631140.5936

\section{RESUMEN}

Los sistemas de gestión surgieron con el fin de apoyar el desarrollo organizacional. Sin embargo, debido a diversidad de criterios que los auditores consideran al momento de su realización, se encontró la necesidad de mejorar las prácticas de auditoría. En este sentido, se requiere orientar a las organizaciones en la gestión de las auditorías con el fin de incrementar la validez y confiabilidad de los datos. Por esto, esta investigación

* Artículo de resultado de investigación.

** Magíster en Gestión de Calidad y Sistemas Integrados de la Universidad Santo Tomás. Ingeniero Civil de la Universidad Santo Tomás. Especialista en Sistemas de Calidad de la Universidad Santo Tomás. Especialista en Seguridad Industrial, Salud Ocupacional y Medio Ambiente de la Universidad Agraria de Colombia. Instituto Colombiano de Normas Técnicas (Icontec). Bogotá, Colombia. Correo electrónico: chaparrojulian@hotmail.com. ORCID: https://orcid.org/0000-0003-1812-5728

${ }^{* * *}$ Magíster en Gestión de Calidad y Sistemas Integrados de la Universidad Santo Tomás. Ingeniero Civil de la Universidad de La Salle. Especialista en Sistemas de Calidad de la Universidad Santo Tomás. Instituto Colombiano de Normas Técnicas (Icontec). Bogotá, Colombia. Correo electrónico: renemar13@hotmail.com. oRCID: https://orcid.org/0000-0002-5585-923X 
presenta una herramienta para la realización de auditorías internas basada en la GTC ISO 19011: 2018 para empresas con sistemas HSEQ. Para ello, se realizó un estudio mixto exploratorio secuencial derivativo. Se diseñó la herramienta con base en las recomendaciones de la literatura y la GTC ISO 19011: 2018 y, posteriormente, se validó su contenido a través de la consulta a expertos. Este proceso indica que el contenido de la herramienta es válido para su aplicación, por lo que puede orientar a empresas con sistemas HSEQ en la gestión de sus auditorías, desde la planeación hasta la generación del informe final y el seguimiento de las acciones derivadas de las no conformidades u oportunidades de mejora detectadas.

Palabras clave: a uditoría, sistemas de gestión de calidad, ambiental, y seguridad y salud en el trabajo.

\section{ABSTRACT}

The management systems were created to support organizational development. However, due to a variety of criteria that auditors consider when conducting an audit, the need to improve audit practices arose. In this sense, organizations required guidance in managing audits in order to increase the validity and reliability of data. That is why this research presents a tool for conducting internal audits based on the GTC ISO 19011: 2018 for companies with HSEQ systems. To this end, a mixed exploratory sequential derivative study was carried out. The tool has been designed based on the literature recommendations and the GTC ISO 19011: 2018 and its content was then validated through expert consultation. The validation process indicates that the tool content is valid for application. The tool can guide companies with HSEQ systems in the management of their audits, from planning to the final report generation and the followup of the actions derived from the non-conformities or improvement opportunities detected.
Keywords: audit, management systems, quality, environmental, health and safety at work.

\section{RESUMO}

Os sistemas de gestão foram criados para suportar 0 desenvolvimento organizacional. No entanto, devido à diversidade de critérios considerados pelos auditores no momento do seu desempenho, foi constatada a necessidade de melhorar as práticas de auditoria. Neste sentido, é necessário nortear as organizações na gestão das auditorias, a fim de aumentar a validade e a fiabilidade dos dados. Consequentemente, esta pesquisa oferece uma ferramenta de auditoria interna baseada no GTC ISO 19011: 2018 para empresas com sistemas de HSEQ. Para esse efeito, foi realizado um estudo exploratório misto sequencial de derivados. A ferramenta foi concebida segundo as recomendações da bibliografia e do GTC ISO 19011: 2018 e o seu conteúdo foi ulteriormente validado através da consulta de peritos. 0 processo de validação indica que o conteúdo da ferramenta é válido para a sua aplicação. A ferramenta pode nortear as empresas com sistemas de HSEQ na gestão das suas auditorias, desde 0 planeamento até à geração do relatório final e ao acompanhamento das ações derivadas das não conformidades ou oportunidades de melhoria detectadas.

Palavras-chave: auditoria, sistemas de gestão, qualidade, ambiental, seguranca e saúde no trabalho.

\section{INTRODUCGIÓN}

Los sistemas de gestión son herramientas que aportan a la productividad y al desarrollo organizacional (Rodríguez-Rojas, 2019). Es este sentido, la Organización Internacional de Normalización (Iso, por sus siglas en inglés) ha desarrollado normas técnicas internacionales en 
diferentes disciplinas (entendidas como temáticas de las normas), entre las que se encuentran la Iso 9001:2015, la ISO 14001:2015 y la ISO 45001:2018. Estos tres sistemas de gestión calidad, ambiental y seguridad y salud en el trabajo tradicionalmente son los más adoptados por las organizaciones.

De acuerdo con el estudio de Ribeiro, Santos, FerreiraRebelo y Silva (2017), la creación de valor en las organizaciones depende de la capacidad de mejorar e innovar continuamente productos y procesos; por lo tanto, se requiere optimizar la gestión al interior de las organizaciones. Para ello, es preciso integrar los sistemas de gestión, con el fin de incrementar el rendimiento y la competitividad de las empresas, reducir la duplicación de tareas, ordenar la documentación, eliminar roles y estructuras que complejizan la gestión, reducir los tiempos y costos de la auditoría y mejorar el desempeño de los sistemas de gestión (Santos, Ferreira-Rebelo, Doiro, \& Santos, 2017; Domingues, Sampaio, \& Arezes, 2016).

En el estudio de Nunhes, Bernardo y Oliveira (2019) se plantean seis principios de integración, uno de los cuales es la estandarización que, a su vez, considera los procesos que se pueden estandarizar elaborando procedimientos que describen cómo debe realizarse una operación 0 tarea determinada. Entre los procedimientos que se pueden integrar están la política, la auditoría interna, el control de seguimiento, los equipos de medición, las acciones correctivas, la selección y evaluación de proveedores, la formación y capacitación, la comunicación, entre otros. En el 2012, se creó la estructura de alto nivel descrita en el Anexo sL de Directiva, Parte 1 de Iso/ IEC, que brinda elementos comunes de los sistemas de gestión, con el fin de armonizar los requisitos asociados a diferentes normas a través de 10 capítulos.

El concepto de integración de los procesos de auditoría en las organizaciones no es reciente, pues se ha trabajado desde hace más de tres décadas. En el campo de los sistemas de gestión de la calidad se contaba con lineamientos para realizar auditorías Iso 10011-1:1990 (lineamientos para hacer auditorías), Iso 10011-2:1991 (criterios para la calificación de auditores) e ISO 100113:1991 (guía para la administración de programas de auditoría). En el campo de los sistemas de gestión ambiental, se contaba con la Iso 14010:1996 (principios generales para auditorías ambientales) y la Iso 14011:1996 (auditorías de sistemas de administración ambiental). De igual manera, los sistemas de gestión de seguridad y salud ocupacional disponían de criterios de auditoría descritos en la OHSAS 18003.

Atendiendo a la diversidad de criterios, requisitos y normas que se gestaron para la realización de auditorías, la ISO, a través de sus comités ISO TC 176 (calidad) e ISO TC 207 (ambiente), crearon las directrices para auditoría de los sistemas de gestión, que fueron consolidadas en la ISo 19011. Esta norma incluyó cuatro aspectos clave: 1) principios de auditoría, 2) gestión del programa de auditoría, 3) actividad de auditoría y 4) competencia y evaluación de los auditores. Su primera versión fue generada en el 2002 y la segunda, en el 2011. En otras palabras, la primera versión de la Iso 19011:2002 anuló y reemplazó a las normas ISo 10011-1:1990, ISO 100112:1991, Iso 10011-3:1991, ISO 14010:1996, ISO 14011:1996 e ISO 14012:1996.

Posteriormente, en el 2018 se generó la versión que actualmente se encuentra vigente, que incluye el enfoque basado en riesgos y riesgos del programa de auditoría, más los requisitos asociados a la competencia de los auditores, asícomo la ampliación del Anexo A de la norma, en el que se brinda orientación adicional destinada a los auditores que planifican y realizan las auditorías, además de la precisión conceptual dada en la actualización de la ISO 9000:2015.

En relación con los avances investigativos en el área de auditoría se evidencian estudios como el de Karapetrovic y Willborn (1998) precisan la necesidad de integrar los procesos de auditoría interna como una estrategia para 
reducir costos. Estos autores señalan que el futuro de las auditorías de primera, segunda y tercera parte es integrarse, dado que favorecen la mejora general de los sistemas de gestión, así como permiten una mejor asignación y despliegue del recurso humano, de los procesos de comunicación y, a la vez, propician la resolución de problemas desde una visión integrada, lo que incrementará la eficacia y eficiencia de los sistemas interconectados.

Karapetrovic (2002) mencionó que al final del siglo XX se incrementó la presión por parte de la industria para armonizar e integrar los sistemas de gestión de la calidad y de gestión ambiental. Sin embargo, reconoce que el proceso de normalización es lento y proyecta la creación de una guía para la implementación de un sistema integrado de gestión; además, el autor, particularmente en el ámbito de la auditoría, indica que la Iso 19011 es el primer paso para tener un enfoque de auditoría integrada que dé cuenta del desempeño organizacional. Karapetrovic (2002) propone un modelo universal de auditoría para la combinación 0 integración de estas auditorías en el ámbito de los sistemas de gestión de la calidad y de la gestión ambiental.

La calidad de la auditoría y las consecuencias en los costos de las auditorías conjuntas se han discutido continuamente(Haak, Muraz, \&Zieseniß, 2018). En particular, la Comunidad Europea en el 2010 generó el libro verde, que contiene la política de auditoría aplicable a esta región. Por otra parte, se han desarrollado modelos conceptuales para comprender los factores que afectan la validez y la confiabilidad de la auditoría en sistemas de gestión particulares, como el de seguridad y salud en el trabajo. Por ejemplo, el planteado por Robson, Macdonald, Gray, Van Eerd y Bigelow (2012) indica cómo los resultados de la auditoría se ven afectados por las características del método de auditoría, el auditor, los lugares de trabajo, el programa de auditoría y el entorno externo. Estos autores concluyen que las recomendaciones brindadas son relevantes tanto para los sistemas de gestión de seguridad y salud en el trabajo como para los de calidad y ambiental.
En términos generales, se evidencia en la revisión de antecedentes que la investigación en el campo de auditoría se ha limitado a reconocer beneficios y falencias del proceso, y que son escasos los estudios que brindan recomendaciones, instrumentos, herramientas o metodologías para facilitar el proceso de auditorías internas en las organizaciones. Aunque se han desarrollado diversas investigaciones que ratifican que la auditoría interna es uno de los procesos que se pueden estandarizar e integrar, la realidad de las organizaciones es que desconocen cómo hacerlo.

Otro aspecto crítico dentro de los procesos de auditoría es que el auditor cumpla con las competencias y expectativas requeridas por la organización objeto de evaluación, dado que a partir de su observación, evaluación de cumplimiento de los requisitos y generación del informe de auditoría - que precisa los hallazgos (no conformidades, observaciones y aspectos por mejorar)—, la alta dirección y los líderes de los sistemas de gestión toman decisiones frente a las acciones correctivas y de mejora del sistema. De acuerdo con Funnell, Wade y Jupe (2016), la utilidad de la auditoría se relaciona principalmente con la capacidad de los informes de auditoría para mejorar la eficiencia operativa de los auditados.

Aunque se cuenta con una norma técnica internacional ISO 19011 que brinda directrices para la auditoría de los sistemas de gestión, se ha detectado que los auditores cuentan con diversas prácticas y criterios al momento de hacer la auditoría, aspecto que reduce la confiabilidad de estas como se demuestra en la investigación adelantada por Robson et al. (2012), quienes señalan que se hay discrepancias entre las prácticas de auditoría reales y las normas internacionales sobre auditoría de sistemas de gestión.

De acuerdo con el estudio de Abreo-Rojas y PinzónRodríguez (2017), se analizaron los informes de las auditorías de tercera parte realizadas a empresas colombianas. En esta investigación se encontró que en los 
sistemas de gestión los aspectos con mayor debilidad son los siguientes: en sistemas de gestión de calidad, auditorías internas, competencia, formación y toma de conciencia, control de los documentos, e infraestructura; en sistemas de gestión ambiental, control operacional, aspectos ambientales y requisitos legales, otros y mejora continua; en sistemas de gestión de seguridad y salud ocupacional (OHSAS 18001), control operacional, identificación de peligros, valoración de riesgos y determinación de los controles, preparación y respuesta ante emergencias, competencia, formación y toma de conciencia, no conformidad, acción correctiva-acción preventiva, medición y seguimiento del desempeño, y auditoría interna. Esto indica que son reiterativas las fallas en los procesos de auditoría interna de en las organizaciones analizadas.

Según Nunhes et al. (2019), es recomendable que las organizaciones que desean integrar sus sistemas de gestión realicen auditorías y capacitaciones de los colaboradores de manera integrada, de modo que los colaboradores puedan articularse con el sistema de gestión, así como comprender su estructura y funcionamiento de manera plena. De acuerdo con lo planteado por Simon, Yaya, Karapetrovic y Casadesús (2014), las auditorías internas integradas son más efectivas, ya que involucran un solo equipo de auditoría, con un solo plan de auditoría y un solo informe final, que pueden contener no conformidades específicas y oportunidades de mejora tanto para cada sistema de gestión como para el sistema integrado.

Además, es importante planificar un programa de auditoría interna que establezca la frecuencia con la que se llevarán a cabo el alcance y los criterios de selección de los auditores, asegurando que las auditorías se realizarán de manera imparcial y que sus resultados serán tratados por la alta gerencia, para que se toman las medidas correctivas apropiadas. Por consiguiente, es esencial contar con herramientas que faciliten tanto la planeación como la realización de las auditorías y que la información derivada de este proceso sea válida y confiable, de tal modo que genere valor agregado en la toma de decisiones.
En Latinoamérica, Colombia y Brasil son los países con mayor número de empresas certificadas, lo que demuestra la necesidad de fortalecer la cultura de auditorías confiables que permitan evidenciar el desempeño real de los sistemas de gestión frente al cumplimiento de sus objetivos y el aporte a la gestión organizacional. De acuerdo con los datos de Iso Survey ${ }^{1}$, Colombia al 2017 contaba con 11.471 organizaciones con certificación en ISO 9001 y 2.954 con certificación en ISO 14001. Los principales sectores certificados son producción de metales, otros servicios, equipos eléctricos y ópticos, transporte, repuestos de vehículos, plásticos, construcción, químicos, maquinaria y equipos y educación.

Atendiendo a este panorama, se requiere brindar a las organizaciones colombianas que cuenten con sistemas de integrados de gestión, también conocidos como sistemas HSEQ, herramientas que faciliten los procesos de auditoría interna en sus sistemas de gestión, considerando la GTC ISO 190011:2018.

\section{METODOLOGÍA}

Este estudio corresponde a una investigación mixta exploratoria secuencial derivativa, que considera dos etapas: la primera corresponde a la revisión de literatura y de la GTC ISo 19011:2018 como base para la construcción de una herramienta para la realización de auditorías internas basada en la GTC ISO 19011:2018 para empresas con sistemas HSEQ; la segunda se refiere a la validación de contenido de la herramienta propuesta a través de la consulta de expertos.

Para la validación de contenido, se efectuó invitación directa a nueve expertos que se relacionan en la tabla 1. Estos fueron seleccionados de acuerdo con los siguientes criterios de inclusión: 1) que contaran con experiencia en procesos de auditoría usando el referente Iso 19011

1 https://www.iso.org/the-iso-survey.html 
y 2) que contaran con formación de pregrado o posgrado relacionado con sistemas de gestión. Se utilizó un cuestionario tipo Likert para el proceso de evaluación.
Este cuestionario fue adaptado de la propuesta de Rodríguez-Rojas (2017). Se consideraron tres dimensiones: claridad, pertinencia y aplicabilidad del instrumento, que fueron indagadas por medio de 14 ítems.

Tabla 1. Perfil detallado de los expertos participantes en la validación de contenido de la herramienta.

\begin{tabular}{|c|c|c|}
\hline \multicolumn{3}{|c|}{ Perfil de los expertos } \\
\hline $\mathrm{N}^{\circ}$ & Formación & Experiencia profesional \\
\hline 1 & $\begin{array}{l}\text { Ingeniero Químico } \\
\text { Especialista en sistemas de gestión integrada }\end{array}$ & $\begin{array}{l}31 \text { años con el Icontec, en el cargo de normalizador, auditor en } \\
\text { los esquemas de calidad y ambiente. }\end{array}$ \\
\hline 2 & $\begin{array}{l}\text { Administrador de Empresas } \\
\text { Especialista en Gerencia de la Calidad } \\
\text { Candidato a Magíster en Calidad y Gestión Integral }\end{array}$ & $\begin{array}{l}\text { Auditor calificado en las normas ISO 9001:2015, OHSAS 18001:2007, } \\
\text { NTC 45001:2018, 6001:2017, NTC 6072:2014. Evaluador de } \\
\text { procesos y servicios para Secretarías de Educación. } \\
\text { Calificación en NTC ISo 14001:2015. } \\
\text { Experiencia de seis años realizando auditorías de tercera parte. } \\
\text { Experiencia en implementación y gestión de sistemas y } \\
\text { requisitos de habilitación para IPS de primer nivel e Instituciones } \\
\text { de Educación. }\end{array}$ \\
\hline 3 & $\begin{array}{l}\text { Ingeniero civil } \\
\text { Especialista en Saneamiento ambiental }\end{array}$ & 14 años como auditor de sistemas de gestión \\
\hline 4 & $\begin{array}{l}\text { Ingeniera industrial } \\
\text { Magíster en Administración de empresas con énfasis en sistemas } \\
\text { integrados } \\
\text { Especialista en Seguridad y salud en el trabajo (en proceso de } \\
\text { titulación) }\end{array}$ & $\begin{array}{l}17 \text { años en procesos de implementación de sistemas de gestión y } \\
\text { desarrollo de auditorías de primera parte, entre los cuales } 5 \text { han } \\
\text { sido realizando auditorías de tercera parte. }\end{array}$ \\
\hline 5 & $\begin{array}{l}\text { Ingeniero químico } \\
\text { Especialista en Gerencia de sistemas de gestión de la calidad, gestión } \\
\text { integrada en calidad, ambiente, riesgos laborales y dirección de } \\
\text { empresas } \\
\text { MBA en Dirección de Empresas }\end{array}$ & $\begin{array}{l}\text { Más de } 30 \text { años como ingeniero, más de } 25 \text { años como auditor de } \\
\text { sistemas de gestión, calificación como auditor de tercera parte } \\
\text { con ISO 9001, ISO 14001, OHSAS 18001/ISO 45001, ISO 22000, QS } \\
\text { 9000, ISO 50001 }\end{array}$ \\
\hline 6 & $\begin{array}{l}\text { Ingeniero industrial } \\
\text { Magíster en Administración estratégica de empresas } \\
\text { Especialista en Higiene y salud ocupacional } \\
\text { Especialista en Sistemas de gestión ambiental } \\
\text { Auditor Líder Icontec en ISO 9001:2015, Iso 14001:2015, ISO } \\
\text { 45001:2018, OHSAS 18001:2007. }\end{array}$ & $\begin{array}{l}\text { Experiencia profesional de } 22 \text { años en empresas tanto de } \\
\text { servicios como de manufactura, liderando el establecimiento, } \\
\text { implementación, mantenimiento y mejora de sistemas de gestión } \\
\text { de calidad, ambiente, seguridad y salud, seguridad vial, gestión } \\
\text { de energía, gestión de activos y continuidad del negocio. }\end{array}$ \\
\hline 7 & $\begin{array}{l}\text { Ingeniero Mecánico (Universidad de América). } \\
\text { Especialista en gerencia de sistemas de gestión de la calidad } \\
\text { (Universidad Santo Tomás) } \\
\text { Especialista en seguridad y salud en el trabajo (ECCI) }\end{array}$ & $\begin{array}{l}\text { Norma ISO } 14001 \text { ( } 800 \text { horas en auditorías de otorgamiento, } \\
\text { seguimiento y renovación) } \\
\text { Norma ISO } 9001 \text { ( } 2.200 \text { horas en auditorías de otorgamiento, } \\
\text { seguimiento y renovación) } \\
\text { Norma OHSAS } 18001 \text { (1.200 horas en auditorías de otorgamiento, } \\
\text { seguimiento y renovación) }\end{array}$ \\
\hline
\end{tabular}




\begin{tabular}{|c|c|c|}
\hline \multicolumn{3}{|c|}{ Perfil de los expertos } \\
\hline $\mathrm{N}^{\circ}$ & Formación & Experiencia profesional \\
\hline 8 & $\begin{array}{l}\text { Ingeniero Civil } \\
\text { Especialista en Gestión Ambiental } \\
\text { Especialista en Construcción Sostenible }\end{array}$ & $\begin{array}{l}\text { Consultoría y auditoría de calidad, ambiental, seguridad } \\
\text { industrial y salud ocupacional, de acuerdo con los lineamientos } \\
\text { de las normas ISO 9001, Iso 14001, oHSAs } 18001 \text { e Iso 45001, desde } \\
1992 \text { a la fecha. } \\
\text { Vinculación laboral con Chrysler Colmotores, 1978, interventoría } \\
\text { de obras civiles. } \\
\text { Industrias e Inversiones Samper S.A., 1979-1981, interventoría } \\
\text { de obras civiles. } \\
\text { Central de Mezclas S.A., 1981-1988, dirección, control y } \\
\text { coordinación administrativa, técnica, productiva y control de } \\
\text { calidad en las plantas de producción de concreto; } \\
\text { Industrias e Inversiones Samper S.A., 1988-1992, dirección } \\
\text { administrativa, técnica y de producción de la Planta de Cemento. }\end{array}$ \\
\hline 9 & $\begin{array}{l}\text { Ingeniera Civil - Especialista en Salud Ocupacional y Ambiental } \\
\text { Magister en calidad }\end{array}$ & $\begin{array}{l}18 \text { años de experiencia en construcción, interventoría y } \\
\text { consultoría en obras civiles. } 12 \text { años como profesional de } \\
\text { certificación Icontec (ISO 9001, oHsAs 18001, Iso 14001, Iso 45001, } \\
\text { entre otros) }\end{array}$ \\
\hline
\end{tabular}

Fuente: elaboración propia.

Con el fin de establecer la validez del contenido y la afinidad de los criterios presentados con los aportados por parte de los expertos, se aplica el método de alfa de Cronbach y W de Kendall, calculados por medio de la formulación en una hoja de cálculo desarrollada por Hernández y Parra (2018).

El alfa de Cronbach permite cuantificar el nivel de consistencia interna de una escala de medida. Se establece un resultado bajo el rango entre 0 y 1 , donde el valor 0 indica que no es confiable y un resultado 1 indica que es totalmente confiable; el valor de aceptación de confiabilidad es de 0,8 (Hernández-Sampieri \& Mendoza, 2018).

Debido a que las variables evaluadas por los expertos son de tipo ordinal de naturaleza no paramétrica, como complemento de la validación se calcula el coeficiente de concordancia W de Kendall. Este coeficiente permite confirmar que el grado de acuerdo y el nivel de concordancia entre los evaluadores expertos que participaron en la revisión del instrumento es muy alto, teniendo en cuenta que cuando el valor es 1 significa una concordancia de acuerdos total (Hernández-Sampieri \& Mendoza, 2018). Una vez obtenidos los resultados de la consulta a expertos, se incorporaron los ajustes en la herramienta propuesta.

\section{RESULTADOS Y DISCUSIÓN}

La herramienta resultado de la investigación se compone de dos instrumentos desarrollados en hojas de cálculo de Excel: la primera detalla el programa y plan de una auditoría interna, mientras que la segunda se concentra en la ejecución de la auditoría. La construcción de la herramienta en conjunto se presenta como el resultado del conocimiento, la experiencia y la sistematización de los autores quienes, luego de establecer los aspectos clave en un proceso de auditoría interna y con el fin de que la herramienta sea de fácil comprensión y genere un impacto positivo en los equipos auditores, , resuelven que debe ceñirse a la estructura de alto nivel creada por ISO y apoyarse en la metodología PHVA. 
El primer instrumento se centra en la planificación y el control administrativo de la auditoría, generando resultados que sirven de apoyo a la ejecución y la presentación de los resultados, y abordando elementos como los datos básicos de auditoría, objetivos y riesgos, elaboración del programa, plan de auditoría, ejecución de la auditoría y normas de consulta, lo que permite organizar previamente la auditoría.

El segundo instrumento, que se centra en la ejecución de la auditoría, recoge algunos resultados del instrumento de planificación para presentar una matriz basada en la estructura de alto nivel, estableciendo ocho categorías: generalidades, liderazgo, apoyo, operación, desempeño, otros, ayuda memoria y normas. Dentro de dichas categorías se encuentran los ítems específicos objeto de validación dentro de una auditoría interna, lo que permite al auditor o equipo auditor cubrir con la gran cantidad de requisitos que conlleva un sistema integrado.

Para poder desarrollar la validación por parte del panel de expertos, lo primero que se realizó fue la consolidación de los resultados en una matriz que permitiera realizar el tratamiento, con el fin de poder analizarlos; adicionalmente, se recogen las observaciones y sugerencias de los expertos en cuanto a la herramienta. En relación con las observaciones, se evidencian diversas diferencias entre los expertos, sobre todo en lo referente a la facilidad de manejo de la herramienta, mientras algunos la consideran adecuada y pertinente con apreciaciones tales como la siguiente: "Es un instrumento novedoso y práctico que facilita las actividades". Otros se inclinan hacia la dificultad del manejo de la información dentro de esta por su volumen, aunque reconocieron abiertamente la utilidad de la herramienta.

La concordancia entre los ítems de cada uno de los criterios establecidos (claridad, pertinencia y aplicabilidad) se establece mediante el coeficiente de Kendall, para lo cual es necesario conocer la cantidad de repeticiones que existen en cada una de las respuestas; en otras palabras, se establece la frecuencia de cada nivel de respuesta por ítem, consolidado dentro de cada categoría obteniendo un X2 que debe ser comparado con un X2 obtenido mediante la tabla de chi-cuadrado, con el fin de aceptar cada uno de los criterios (si el X2 calculado es menor al X2 de la tabla, entonces es aceptado el criterio), para cuyo caso se utilizará un margen de confianza del 95\%. Los resultados se evidencian en la tabla 1 .

Tabla 2. Coeficiente de concordancia W de Kendall

\begin{tabular}{|l|c|c|c|}
\hline \multicolumn{1}{|c|}{ Datos / Criterios } & Claridad & Pertinencia & Aplicabilidad \\
\hline $\mathrm{T}$ & 139 & 168 & 169 \\
\hline $\mathrm{U}$ & 4.837 & 5.666 & 5.725 \\
\hline $\mathrm{N}$ & 4 & 5 & 5 \\
\hline $\mathrm{M}$ & 8 & 8 & 8 \\
\hline $\mathrm{S}$ & 6,75 & 21,2 & 12,8 \\
\hline $\mathrm{W}$ & 0,064903846 & 0,055789474 & 0,035555556 \\
\hline $\boldsymbol{\chi}^{2}$ calculada & 1,557692308 & 1,785263158 & 1,137777778 \\
\hline $\mathrm{V}$ & 21 & 28 & 28 \\
\hline $\boldsymbol{\alpha} \approx \mathrm{P}$ & 0,05 & 0,05 & 0,05 \\
\hline $\boldsymbol{\chi}^{2}$ Tabla & 11,591 & 16,928 & 16,928 \\
\hline Aceptación Hipótesis & Ha: Aceptación criterio & Ha: Aceptación criterio & Ha: Aceptación criterio \\
\hline
\end{tabular}


De igual manera se realiza el cálculo del alpha de Cronbach (tabla 2), cuyo resultado es de un valor de 0,9060, lo que indica una alta fiabilidad en la escala usada y determina que existe una independencia fuerte entre los 14 ítems evaluados.

Tabla 3. Alfa de Cronbach

\begin{tabular}{|l|c|l|}
\hline \multicolumn{3}{|c|}{ Alfa de Cronbach - Convenciones } \\
\hline K: & 14 & El número de ítems \\
\hline$\Sigma \mathrm{Si}^{2}:$ & 31,01098901 & Sumatoria de las varianzas de los ítems \\
\hline $\mathrm{S}_{\mathrm{T}}{ }^{2}:$ & 195,4285714 & La varianza de la suma de los ítems \\
\hline$\alpha:$ & 0,906034811 & Coeficiente de alfa de Cronbach \\
\hline
\end{tabular}

Fuente: elaboración propia.

A partir de los resultados, se puede establecer que existe una concordancia significativa entre los rangos asignados por los expertos; sin embargo, la no proximidad del factor w a 1 determina que la fuerza de concordancia no es tan fuerte. En conclusión, se determina que la herramienta desarrollada tiene una validez de contenido teniendo en cuenta los resultados de Cronbach y Kendall.

\section{CONCLUSIONES}

Las auditorías internas requieren de un proceso ordenado y basado en evidencias y criterios objetivos para garantizar la validez y confiabilidad de los datos, tal y como lo refieren los estudios analizados. Este proceso debe organizarse bajo la lógica del ciclo PHA y bajo un enfoque de riesgos y oportunidades de la gestión. Asimismo, los objetivos de la auditoría deben ser coherentes con las necesidades y expectativas de las partes interesadas: las características y los requisitos de los procesos, productos, servicios y proyectos, y cualquier cambio en ellos; los requisitos de sistema de gestión; la necesidad de evaluar a los proveedores externos; el nivel de madurez de los sistemas de gestión (KPI); la ocurrencia de no conformidades o incidentes o quejas de las partes interesadas; los riesgos y oportunidades identificados para el auditado, y los resultados de las auditorías previas.

La falta de uniformidad en la formación y experiencia de los auditores es una de las grandes dificultades generadas en torno a los procesos de auditoría; de ahí que la necesidad de diseñar e implementar herramientas y guías permitan fortalecer este proceso, no solo desde el punto de vista operativo, sino desde la planificación y hasta la entrega de resultados; más aún, debe generar estrategias de seguimiento posterior. Adicionalmente, en los modelos de auditoría de sistemas integrados se presenta la dificultad del manejo de una gran y variada cantidad de información, por lo que también se hace necesario contar con información guía que sea puntual y de fácil manejo.

Abordar el proceso de auditoría definido en dos momentos - planificación y ejecución - facilita el control de la auditoría al permitir asignar roles, momentos y responsabilidades de una manera estructurada en un momento previo a la auditoría; además, en el momento de ejecución se puede traer dicha información siempre que sea pertinente, momento en el que es importante tratar de garantizar que los criterios se encuentren ceñidos a los requerimientos de la norma Iso 19011, enfocada en la estructura de alto nivel y manteniendo la estructura del PHVA.

La herramienta resultante, compuesta por los dos instrumentos mencionados, tuvo una buena acogida por parte de ocho expertos, quienes concordaron en general en afirmar que permitiría reducir tiempos al organizar de una manera sistémica los pasos y procesos correspondientes a una auditoría interna. Igualmente, al realizarse la validación de contenido de las respuestas por medio del alpha de Cronbach, cuyo valor fue de 0,95 , se valida la escala de medición; por otra parte, se hizo uso del coeficiente de Kendall por cada dimensión evaluada (claridad, pertinencia y aplicabilidad), con el que se 
obtuvo como resultado que no existe interdependencia marcada entre los ítems que conforman cada categoría, avalando de esta manera el instrumento.

\section{REFERENCIAS}

Abreo-Rojas, N., \& Pinzón-Rodríguez, N. (2017). Guía para la implementación de NTC ISO 9001:2008, NTC ISO 14001:2004 y NTC OHSAS 18001:2007, basada en los hallazgos de las auditorías de certificación realizadas por el Icontec entre junio de 2012 y junio de 2015. SIGNOS - Investigación en Sistemas de Gestion, 9(2), 149-158. DoI: https://doi.org/10.15332/s21451389.2017.0002.09

Domingues, P., Sampaio, P., \& Arezes, P. M. (2016). Integrated management systems assessment: A maturity model proposal, Journal of Cleaner Production, 210, 977-993. DoI: https://doi.org/10.1016/j.jclepro.2016.02.103

Funnell, W., Wade, M., \& Jupe, R. (2016). Stakeholder perceptions of performance audit credibility, Accounting and Business Research, 46(6), 601-619. DOI: https://doi.org/10.1080/00014788.2016.1157680

Haak, M., Muraz, M., \& Zieseniß, R. (2018). Joint Audits: Does the Allocation of Audit Work Affect Audit Quality and Audit Fees? Accounting in Europe, 15(1), 55-80. DoI: https://doi.org/10.1080/17449480. 2018.1440611

Hernández, H., \& Parra, J. (2018). Instrumento para medir el nivel de integración de los sistemas de gestión en organizaciones colombianas (Trabajo de grado de maestría). Universidad Santo Tomás, Bogotá, Colombia.

Hernández-Sampieri, R., \& Mendoza, C. (2018). Metodología de la investigación. Las rutas cuantitativa, cualitativa y mixta. Ciudad de México: McGraw Hill. DoI: https://doi.org/10.17993/CcyLl.2018.15
International Organization for Standardization. (2015a). ISO 9001:2015. Sistema de gestión de la calidad. Requisitos. Suiza: ISO.

International Organization for Standardization. (2015b). ISO 14001:2015. Sistema de gestión ambiental. Suiza: ISO.

International Organization for Standardization. (2018a). ISO 45001:2018. Sistemas de gestion de la seguridady salud en el trabajo - Requisitos con orientación para su uso. Suiza: ISO.

International Organization for Standardization. (2018b). ISO 19011:2018. Directrices para la auditoría de los sistemas de gestión. Suiza: ISO.

Karapetrovic, S., \& Willborn, W. (1998). Integration of quality and environmental management systems. The TQM Magazine, 10(3), 204-213. Dor: https://doi. org/10.1108/09544789810214800

Karapetrovic, S. (2002). On the concept of a universal audit of quality and environmental management systems. Corporate Social Responsibility and Environmental Management, 9(3), 147-156. DoI: 10.1002/ csr. 16

Nunhes, T. V., Bernardo, M., \& Oliveira, O. J. (2019). Guiding principles of integrated management systems: Towards unifying a starting point for researchers and practitioners. Journal of Cleaner Production, 210, 977-993. DoI: https://doi.org/10.1016/j. jclepro.2018.11.066

Ribeiro, F., Santos, G., Ferreira-Rebelo, M., \& Silva, R. (2017). Integrated management systems: Trends for portugal in the 2025 horizon. Procedia Manufacturing, 13, 1191-1198. DoI: https://doi.org/10.1016/j. promfg.2017.09.194

Robson, L. S., Macdonald, S., Gray, G. C., Van Eerd, D. L., \& Bigelow, P. L. (2012). A descriptive study of the oHs management auditing methods used by 
public sector organizations conducting audits of workplaces: Implications for audit reliability and validity. Safety Science, 50(2), 181-189. Dor: https:// doi.org/10.1016/j.ssci.2011.08.006

Rodríguez-Rojas, Y. (2019). La gestión integral como una herramienta de la productividad. SIGNOS - Investigación en Sistemas de Gestión, 11(1), 11-13. DOI: https://doi.org/10.15332/s2145-1389-4984

Rodríguez-Rojas, Y., \&Pedraza-Nájar, X. (2017). Aportes de la estructura de alto nivel en la gestión integrada. Revista Global de Negocios, 5(2), 65-75.

Rodríguez-Rojas, Y. (2017). Evaluación de la madurez de la gestión de la seguridad y salud en el trabajo en universidades con acreditación de alta calidad multicampus de Bogotá(Disertación doctoral). Universidad de Celaya, Celaya, México.

Santos, D., Ferreira-Rebelo, M., Doiro, M., \& Santos, G. (2017). The integration of certified management systems. Case study - organizations located at the district of Braga, Portugal. Procedia Manufacturing, 13, 964-971. DoI: https://doi.org/10.1016/j. promfg.2017.09.168

Simon, A., Yaya, L. H. P., Karapetrovic, S., \& Casadesús, M. (2014). An empirical analysis of the integration of internal and external management system audits. Journal of Cleaner Production, 66, 499-506. DoI: https://doi.org/10.1016/j.jclepro.2013.11.020 